\title{
Mujeres entre la enseñanza en las escuelas y la escritura sobre las prácticas educativas. Labor de maestras argentinas de los primeros decenios del 1900
}

Women between teaching in schools, and writing about educational practices. The work of some Argentine teachers of the early 1900s

Agustina Mosso

DOI: https://doi.org/10.24215/25457284e123

Investigaciones Socio-históricas Regionales, Centro

Cientifico Tecnológico Rosario, CONICET, Argentina

agustina_mosso@hotmai.com

Recepción: 14 Marzo 2018

Aprobación: 07 Mayo 2019

\section{Resumen:}

El corpus documental examinado en el presente trabajo lo constituyen libros de texto para el aula, cuyas autoras son maestras. $\mathrm{El}$ análisis de estas obras, publicadas en las primeras décadas del siglo XX, da lugar a reflexiones acerca del vínculo maestrasalfabetización-escritura. Al adentrarnos en la minuciosa lectura del contenido de las publicaciones (que incluye índices, sumarios, guías, palabras preliminares, advertencias, presentaciones) elaboramos un conjunto de ideas y juicios que reflexionan sobre maestras, junto al proceso de feminización de la docencia, como productoras de saberes e intelectuales.

Palabras clave: Mujeres, Escritura, Docencia, Inicios Siglo XX.

\section{Abstract:}

The documentary corpus examined is school books whose authors are teachers. The analysis of these works, published in the first decades of the twentieth century, provides reflections on the teacher-literacy- writing. With the reading of the content of the publications (which includes indexes, summaries, guides, preliminary words, warnings, presentations) we develop ideas and judgments that reflect on teachers, in the process of feminization of teaching, as producers of knowledge and intellectuals.

KEYWORDS: Women, Writing, Teaching, Early 20th Century.

\section{INTRODUCCIÓN}

El presente escrito reúne los primeros avances de una pesquisa que indaga en torno a maestras que, además de ejercer en el aula, escribieron libros de texto para sus alumnos en los inicios del 1900 en Argentina. Hemos encontrado numerosas pruebas que permiten pensar que las maestras, como agentes alfabetizadas, complejizaron las prácticas de enseñanza en el aula escribiendo materiales para sus alumnos, entre los cuales podemos mencionar libros de lectura o cartillas para alfabetizar. Aquí trabajaremos con libros de texto a modo de "territorios de imposición simbólica e institucionalización de las relaciones entre saber y poder en la escuela” (Martínez Bontafé, citado en Sardi, 2010, p. 91). Analizaremos la participación de maestras por fuera de su labor de enseñanza en las aulas, en una tarea muy relacionada a dicho trabajo pero que implica otras destrezas. Conjeturamos que dicho trabajo las habilitó a compartir e intercambiar sus pensamientos con diversos actores del ámbito de lo público, en los cuales no era usual su desempeño, y con quienes compartieron y discutieron asuntos culturales y educativos. Fueron estas maestras quienes lograron articular demandas del ámbito de la enseñanza a través de escritos que dan cuenta del compromiso que contrajeron con su labor educativa. 


\section{Breve ConteXtualización Histórica}

Ubicamos en la segunda mitad del siglo XIX discusiones acerca de si era conveniente incorporar o no a las mujeres al ejercicio del magisterio. Ellas fueron colocadas tiempo después, en las primeras décadas del siglo XX, frente a trabajos relacionados con la tarea de enseñar. Y esto sucedió de un modo particular: las mujeres se convertirían en segundas madres, protectoras del hogar y de la escuela. A su vez, y con la Ley de Educación Común No1420, sancionada el 8 de julio de 1884, encontramos los "frutos del accionar sarmientino" (Solari, 1991, p.187). Con sus principios fundamentales (obligatoriedad, gratuidad, gradualidad y laicidad), entendemos la aparición de la base para la organización de la escuela primaria argentina. En ese momento, prevalecían consideraciones materiales que, junto a la percepción de que las mujeres estaban mejor preparadas para encarar la educación primaria, determinaron que el "Estado debiera diseñar políticas con el afán de feminizar el magisterio” (Fiorucci, 2016, p. 122) Será popularizado entonces un discurso sobre la importancia de la madre educadora profesional, que llega a Argentina en el siglo XIX desde Europa y es adaptado a los intereses locales de países recientemente independizados (Yannoulas, 1996). El Estado Nacional, garante del derecho, necesitaba quien ejerciera la profesión docente con motivo de expandir la educación popular, en creación de la base para la homogeneización cultural. Poniendo en marcha un proceso de profesionalización para cubrir las necesidades de una demanda de educación primaria casi plena (que requirió de ocho décadas), conformó a la Escuela Normal en la institución fundamental para la instauración de la educación primaria universal, mientras que universidades y unos pocos institutos especializados cubrieron la formación de profesores secundarios. Los docentes vinieron a conformar un sector diverso, diferenciado por su formación y por las posteriores jerarquías laborales y retribuciones salariales. En este contexto, ingresarán las mujeres al mercado de trabajo. Ellas comenzarán a vivir fenómenos emancipadores como el acceso a más y mejores conocimientos, la circulación libre en espacios públicos y la obtención de un dinero con el cual adquirir posiciones propias. Sin embargo, como señala Flavia Fiorucci, "no se puede negar la flagrante contradicción que suponía hacer a las mujeres responsables de crear al ciudadano mientras que estas eran privadas del derecho a una ciudadanía plena" (2016, p. 123). A pesar de la inferioridad jurídica que poseían las mujeres, advertimos una expansión en los desempeños femeninos dentro de los aspectos educativos y culturales.

Aunque los espacios cedidos a ellas se abrían apenas, la educación fue una zona que las habilitó a circular en espacios públicos que antes les eran vedados. Por otra parte, y como dijimos arriba, con la Ley 1420 (cuya jurisdicción abarcó la Capital Federal y los Territorios Nacionales) se estableció un Consejo Escolar en cada distrito de la campaña y un Consejo Nacional de Educación (Puiggrós, 1996, p.64). Fue este el órgano gubernativo que sancionó en 1877 el Reglamento sobre textos escolares, destinado a "marcar las pautas esenciales" para que las comisiones reunidas al efecto (Alloatti, 2007, p. 95) aprobaran o no los libros de lectura que autores/as y directores presentaban bajo solicitud. A cargo del Estado Nacional quedaba entonces la introducción del sistema escolar primario en todo el país, junto con la edición de libros de textos que subscribía su Consejo, con la finalidad de difundir el aprendizaje de la lectura a todo el público infantil. Otra función primordial del Consejo era la de dirigir una publicación mensual de educación, El Monitor de la Educación Común, revista oficial cuyos escritos se iniciaron en 1881 y cuyas fuentes editadas (de tipo periodístico) han sido elementos decisivos en la cristalización de una imagen prescriptiva o punitiva del currículo, la didáctica y el gobierno de la educación.

Dicho boletín, en la sección oficial, publicaba las actas de sesiones y otros documentos formales. Tanto en esta como en su segunda parte, la que contenía literaturas pedagógicas y didácticas, reseñas bibliográficas de revistas nacionales y extranjeras, notas literarias e históricas, localizamos las recomendaciones de los libros de texto publicados por maestras. En la nómina de textos de lectura aprobados por el Honorable Consejo de Educación leemos los nombres de las docentes, seguidos del título del libro que se recomendaba fuera leído en las escuelas argentinas. 
Precisamente, Clara de Toro y Gómez aparecerá en la edición número 640 del año 1926. En el mes de abril serán recomendados sus libros "El hogar de todos" y "La escuela y la vida". Lo mismo ocurrirá con "Mi librito" (1924) de Delfina Piuma Shmid. Además, en la edición número 678 del año 48, leemos en la Sesión número 99 (del día 19 de octubre de 1928) con el expediente número 20405- M- 1928: "Autorizar la inclusión en la lista de textos aprobados para primer grado superior el libro "Días de sol", de que es autora la señora Evangelina M. de Mercado Vera, de conformidad con lo informado por la Inspección Técnica General”. Lola S. B. de Bourguet, Micaela Iantorno de Nasino, Julia Andrés de Valls, Adelina Méndez Funes de Millán, serán otras maestras que, del mismo modo, aparecerán en el Monitor. Según Adrián Ascolani, "los libros de lectura fueron el principal instrumento material para sostener el discurso civilizador escolar, haciendo las veces de manuales de urbanidad, de decálogos de principios y valores éticos" (Ascolani, 2010, p. 305). En este sentido, estos libros expresaban un imaginario reformista que mixturaba componentes diversos: religiosos, ideológicos y científicos. Incumbía al Consejo disponer que, de $1^{\circ}$ a $3^{\circ}$ grado, el único libro de texto empleado debiera ser el libro de lectura, texto utilizado por el conjunto de los escolares. Fue recién en la década del '20 que maestros y maestras de las escuelas primarias del país podían escoger los libros que luego comunicaban a inspectores, quienes elevaban la notificación al Consejo, garante de repartir gratuitamente ejemplares a las escuelas solicitantes.

Era en los concursos realizados bajo intervención del Consejo Nacional de Educación donde se determinaban los textos que serían utilizados en las escuelas; se convocaba allí a autores/as, editores, introductores de libros a presentar sus solicitudes acompañadas de los ejemplares. En función de este mecanismo, puede pensarse al libro de lectura como instrumento organizador de la práctica escolar, legitimador de los saberes que lo conforman y muestra del currículum activo.

Una de las principales funciones del libro sería la de normalizar, ordenar y sistematizar la población escolar que ingresaba a la escuela y llevaba "el lastre de lo heteróclito y contaminante..." (Sardi, 2010, p. 91). Los espacios privilegiados para postular las diversas concepciones sobre el libro de lectura, su uso y efecto en la escuela fueron los informes ministeriales, los digestos, las revistas pedagógicas -oficiales y contra hegemónicasy los prólogos de los propios libros. Estos documentos funcionaron como territorios de intervención de quienes trabajaban en el magisterio argentino.

Tomando los aportes de la historia con mujeres en perspectiva de género, junto a la historia social y de la educación bajo un registro cultural nos disponemos a indagar sobre la producción de textos escolares realizados por maestras. Para operacionalizar este trabajo nos adentramos en una intensa búsqueda por diversos archivos de la ciudad de Rosario (Biblioteca Argentina, Biblioteca Pedagógica Eudoro Díaz).

Con lo allí relevado, y a los efectos de conformar un corpus de libros de texto a analizar, y ayudados por un enfoque metodológico cualitativo, interpretativo y de lectura crítica de estos documentos escritos (Aróstegui, 1995), realizamos un compendio de la producción editorial de un grupo de educadoras. Asimismo, y partiendo de un supuesto muy propio de la historia sociocultural, nos interrogamos con dicha presunción acerca de la tensión entre las prescripciones y las prácticas (Chartier, 2008). Al sancionarse la Ley No 1420, ${ }^{1}$ en su letra fue establecido un corpus de saberes con el objeto de formar varones y mujeres capaces de ocupar lugares que la sociedad deparaba para cada uno/a. ${ }^{2}$ En este punto, los libros de texto operaron sobre una agenda de corte nacional que invita (en futuras pesquisas) a considerar la singularidad de las prácticas en la inmensidad de una geografía extendida más allá del lugar físico donde estaba emplazada la toma de decisiones acerca de las prescripciones escolares. Los libros aquí estudiados, al igual que su distribución, pertenecían a Buenos Aires ya que las editoriales eran de dicho lugar, pero eran usados por el resto del país, con los rasgos de cada territorio.

En adelante, daremos a conocer el análisis del contenido de libros de maestras. Luego, unas conclusiones darán apertura a nuevas observaciones sobre la temática. 


\section{ENSEÑANZA y ESCRITURA. QUÉ hAY AL INTERIOR DE LOS LIBROS DE LAS MAESTRAS}

Siguiendo los escritos de Dussel y Caruso (1999), podemos considerar a la escuela primaria pública argentina situada dentro de un sistema educativo nacional, integrador y homogeneizador de la población de las provincias (principalmente de la mayoría de inmigrantes), factor clave de la inclusión de Argentina dentro de los países de modernización temprana en Latinoamérica. Escuela primaria acompañada por un sistema uniforme de formación de docentes a quienes, en muchos casos, se convocó en la elaboración de libros escolares. Las maestras que traspasaron la labor educativa, dedicándose a escribir sobre y por la mejora de las prácticas áulicas, fueron mujeres que en su período se animaron a utilizar las herramientas que la formación en el oficio les brindaba para ir más allá de la enseñanza en las escuelas, escribir y publicar. Ellas diagramaron libros cuyo contenido lo conformaban los saberes que, al permanecer prácticos solo en el momento en que fueron aplicados, hoy resulta compleja la tarea de encontrarlos en los archivos y centros documentales. Luego de reiteradas visitas a estas bibliotecas determinamos que sus textos, vinculados en lo íntimo a la experiencia que el trabajo en la docencia brindó a las maestras, eran presentados por sus autoras ante concursos que aprobarán su propagación en las escuelas. Como dijimos, aquellos que el Consejo de Educación certificaba para su distribución.

Por otra parte, y si pensamos en los inicios de la escuela pública argentina como aquella que se presentó a modo de medio de igualación de lo diferente, produciendo en su seno un proceso de "argentinización" de los inmigrantes, podemos afirmar que el libro de texto fue aquel dispositivo de carácter central en la construcción de la identidad nacional (Narodowski y Manolakis, 2001). Los libros de lectura eran un refuerzo a las recomendaciones de conductas adecuadas que el maestro haría continuamente, "siendo su función normativa y preventiva” (Ascolani, 2010, p.319). En ellos aparecían recomendaciones referentes a la relación afectiva intensa que debía suceder con los alumnos, que implicaba muchas veces la feminización y "maternización" de la función docente. De ahí que podamos hallar en búsquedas extensas por bibliotecas, libros de texto para el aula cuya autoría estaba en manos de las propias maestras. A continuación, se describe qué hallamos al interior de los libros.

Bien lo dijimos: las mujeres, certificadas con sus títulos de Maestras Normales, hábiles en el conocimiento y destreza de las prácticas de lecto-escritura, fueron escritoras y autoras de libros. Asimismo, sus obras giraron en torno a la actividad educativa y trascendieron únicamente gracias al reconocimiento que en las escuelas obtuvieron.

Estimamos comenzar con el análisis de los libros partiendo por el más antiguo de lectura para el primer grado superior, denominado Por nuestro bien y escrito por Micaela Iantorno de Nasino. Al igual que los demás escritos aquí estudiados, se encuentra aprobado por el Consejo Nacional de Educación y los editores de dicha publicación son los Talleres Gráficos Cappellano Hermanos, de la ciudad de Buenos Aires. El prólogo está firmado por la autora en dicha metrópoli en 1926, año que coincide con el de la publicación de la obra. Ella allí dice presentar ni "un libro más", "ni obra nueva", sino útil para lograr que el niño salve con mayor facilidad las dificultades que pueden presentársele en el aprendizaje de la lecto-escritura. Se caracteriza por ser beneficioso para los procesos educativos. María Olguín y Virginia Zamora Grondona son las autoras del próximo libro a estudiar: Lucha. Destinado al cuarto grado y aprobado por el Consejo Nacional de Educación bajo el expediente número 15136-E-1927, el ejemplar que poseemos responde a la décima edición de la obra, publicada por Ángel Estrada y Cía. Editores, de Buenos Aires, en 1936. Fue entonces en el mes de enero de 1926 cuando las autoras de la primera edición del libro dejaron en claro dentro del prólogo que con dicha obra pretendían auxiliar a las demás materias que formaban parte del programa de enseñanza, siendo el libro de lectura ayuda para las necesidades intelectuales de los alumnos. Su producción pertenecía a mujeres que poseían largos años en la práctica de la instrucción primaria. Solidarias con quienes a diario trabajaban en la docencia, su obra sería de gran utilidad para los/las docentes. Finalmente, Alcira Del Prado en su texto de lectura para primer grado, Sol, presenta en 1932 un escrito que viene a proponer un método novedoso para 
enseñar a leer y a escribir. Ella considera que la experiencia en las aulas la anima a dar a conocer este libro que coloca en concursos y obtiene diversas aprobaciones: la del Consejo General de Educación de la provincia de Buenos Aires; la del Consejo Nacional de Educación, como así también la aceptación del Consejo General de Educación de las provincias de Santa Fe, Santiago del Estero, Mendoza y San Juan. Contamos con la edición cuarta, referente a la publicación de la editorial porteña Independencia.

Entonces, y si nos adentramos en el contenido de los libros, el de Micaela Iantorno de Nasino refiere al principio: "A los señores Miembros de la Comisión de Textos del Consejo Nacional de Educación". Allí la autora se dirige al final de la introducción con motivo de revalidar su libro y resignificar un trabajo que denomina "de rigurosa coordinación metódica". Es por ello que aguarda que tanto directivos como maestros lo utilicen como texto de lectura para el Primer Grado Superior. Vemos que el índice del escrito se encuentra dividido en cuatro partes; la primera consta también de cuatro secciones. En la número uno aparecen temáticas como "El barrilete", "El niño estudioso", "Un soldadito", "El aseo es el mejor adorno", "Trabajar es un deber". En la sección dos, los títulos "Flores y frutas" (aclara que es para ser usado como tema de conversación), "No debes ser glotón”, "Muertos por la patria", "El perrito travieso", "Piedras preciosas". En la tercera sección, "Deseos de aprender", "Las dos muñecas", "Al aire libre”, "El gatito herido", "El cumpleaños del abuelo", "Algo de historia", "La lección de mamá". Y en la última sección leemos: "Leve accidente", "A cazar", "Lo que vio Elsita", "Narraciones del abuelo", "Poetas argentinos". La parte que sigue presenta también cuatro secciones. La primera menciona "Una imprudencia evitada”, "Oro y cobre", "Primer Gobierno Patrio" (tema de conversación), "Una pequeña lectora". La sección dos posee los títulos: "No se debe mentir", "El progreso en los medios de transporte" (tema de conversación), "La limpieza en todo". La sección tercera dicta el "Globo geográfico", "La imprudencia no es valor", "Ayudemos a los necesitados", "Uno de nuestros héroes". Y la sección que cierra la segunda parte recita: "La zeta no es comestible", "Sembrando trigo" (tema de conversación), "Pequeño pero listo". La tercera parte nos muestra la sección uno que narra "Cielo nublado", "Papel matamosca”. La número dos: "Madre”, "Desobediencia castigada”, "Declaración de la Independencia Argentina”, "Fechas gloriosas”, "Mi bandera”. La penúltima sección: "Sombra y frutas", "Sarmiento", "Los puntos cardinales", "Las estaciones". Y la última sección "Diarios y cartas" (temas de conversación), "Podemos osar, pero no hozar". Finalmente, y ya en la cuarta y póstuma parte, leemos tres secciones de las cuales la número uno habla del "Respeto a los ancianos", "El alcoholista" (tema de conversación), "El atlas del abuelo", "Aire, limpieza y luz". La número dos sobre "Volvieron las golondrinas", "El ombú”, "Nuestro himno nacional”. Y la tres: "En lo alto o en lo profundo", "La hija del vigía", "La escuela", "Yo sé leer". Cada página de Por nuestro bien incluye notas al pie que sirven al alumno para comprender aquellas palabras que quizás desconoce. Iantorno de Nasino decide colocarlas a modo de sugerencia.

Además, el libro despliega poemas en los que se distingue que la autora es quien los escribe porque no presentan una firma al final de los mismos; allí abundan los diálogos en los que aparecen, entre otras cosas, consejos del buen obrar para los niños.

Olguín y Zamora Grondona en cambio, exponen a través del compendio Lucha un sinnúmero de temas concernientes a todas las asignaturas del programa oficial, pero de un modo innovador; por meses y ya no por materias. Los asuntos ordenados de esta forma, pensamos así lo intuyeron las escritoras, facilitan a maestros/as y a alumnos su aplicación. Lucha presenta una entretenida lectura sobre la diversidad de temas que debe trabajar un alumno de cuarto grado: "Moral", "Higiene", "Cívica", "Naturales" (las estaciones del año que transcurren estando el niño en la escuela), "Historia", "Trabajos literarios" (prosas y poesías de autores reconocidos), "Geografía", "Botánica”, "Zoología”, "Mineralogía”, "Fenómenos físicos y químicos". Por ejemplo, si nos centramos en el mes de marzo ubicamos: "Las clases se inician”, "Árboles viejos” (poesía), "Evocando", "Nueva vida", "Guillermo Brown" (historia), "Un pueblo subterráneo" (zoología), "Seamos curiosos" (botánica), "No creamos en las alabanzas" (moral), "Muerte de Florencio Varela” (historia), "La canción de las hojas" (otoño), "Paz es riqueza” (poesía), "Los experimentos de Guillermito” (fenómenos 
químicos), "Una fuente de riqueza". Ya por el mes de julio leemos: "La conquista del aire", "Ignacio Pirovano", "9 de julio" (poesía), "Casas y cosas de otros tiempos" (historia), "Los cabellos de la abuelita", "Fuentes minerales y termales" (mineralogía), "Por qué llegó tarde Marcelito" (instrucción cívica) y "Ahorra”. Ambas maestras presentaron la información de manera ordenada; por ejemplo, entre paréntesis, mostraban la correspondencia entre el título de la temática con la materia que consideraban debía incluir ese tópico. Notamos en este escrito la presentación de un método particular de enseñanza de los contenidos para cuarto grado. En seguimiento del desarrollo de los meses del año, las autoras de Lucha adjuntaban además un índice general que abarcaba desde el prólogo hasta el último tema del mes de noviembre. Como dijimos, contamos con un ejemplar referente a la décima edición (1936), sospechando el éxito de la primera allá por 1926.

"A los maestros", se titula el primer apartado del libro de Alcira Del Prado, Sol, término que utiliza a modo de clave metodológica de la obra, e invita a sus colegas (conocedores de las eventualidades del magisterio, dice ella) a poner en práctica las ejercitaciones que propone dentro del ámbito escolar.

Cierra este título y reafirma los aportes que espera consumar con la publicación de su libro: “...la sinceridad de nuestro propósito, con el que colmamos aquel anhelo de todos los que enseñan con vocación: dar de sí todo al niño, es decir, a la patria" (A. Del Prado, 1932, p.10).

El ordenamiento del libro, al final, aparece de un modo particular. Alcira presenta un detalle de lo narrado con motivo de ser apropiado por el maestro y usado ante la aparición de dudas sobre el desarrollo de los temas. Lo suyo es un índice guía que alude a las páginas que desea referenciar. Ella aclara, por ejemplo, en la hoja 11- Introducción de la palabra generadora sol, que se enseñará como un todo. 12Descomposición de la palabra sol. Sílabas ol y so; letras s-o y l. 13- Afianzamiento de lo enseñado e introducción de la letra de imprenta. Derivadas: lo y solo. 14- Ilustración destinada a fijar la atención del niño, representando una escena: "la recolección de la sal". 19- Afianzamiento de lo enseñado e introducción de nuevos derivados. 20- Recapitulación de lo enseñado y enseñanza de la mayúscula L. 30- Empleo del acento. Ejercitación. Introducción de la I y de la S mayúsculas. 90- Lectura corriente "Los deditos" (Verso) 106- Lectura corriente "Un ayudante que nada pide". Esta publicación utiliza imágenes que acompañan las letras imprentas (minúscula primero, cursiva después), gran variedad de oraciones, diálogos en los que se discriminan preguntas de respuestas, afirmaciones de enunciados, para enseñar a leer y a escribir a los pequeños de primer grado. Con cuentos breves incluidos en las conversaciones, poemas en los cuales los animales cobran vida y opinan sobre sus experiencias, los niños aprenden también sobre el mundo de la economía doméstica del hogar y los quehaceres familiares. Al interior de esta pluralidad de temas leemos instrucciones para el armado de un barrilete, oraciones que procuran que los alumnos se apropien de letras y sílabas, enumeraciones en las que los autores son los propios estudiantes. Distinguimos asimismo fábulas con moralejas, composiciones que pueden ser usadas a modo de canciones para aprender, por ejemplo, la cantidad de días que tienen los meses, rimas, odas y cuentos (tal el caso donde el autor es Amado Nervo) para conocer el calendario.

Arriba descritos los compendios, pasamos entonces a desarrollar unas reflexiones finales que ayudarán a cotejar y comprender la escritura de los libros seleccionados. Pensar en obras cuya autoría pertenece a mujeres vinculadas al magisterio, y siguiendo los escritos de Flavia Fiorucci $(2013$; 2016), reparamos en dos aspectos fundamentales: la docencia como proceso feminizado promovido por el propio Estado argentino y las mujeres destinadas a dicha actividad como maestras creadoras de saberes de aula, competentes para la producción de libros de texto escolares y aptas para participar en el debate sobre la enseñanza, el oficio docente.

\section{Palabras de cierre}

Habiendo realizado a lo largo de la presente comunicación un somero análisis sobre el contenido de libros elaborados por maestras (uno de los cuales es redactado en coautoría y nos conduce a pensar en duplas 
de maestras comprometidas con una labor educativa de creación de saberes, alejadas de la cotidianeidad escolar pero próximas a las problemáticas que de ella surgían e inquietaban a las educadoras a escribir sobre las mismas), publicados y aprobados por el Consejo de Educación, nos encontramos en condiciones de consolidar unos acercamientos al tema estudiado que puntualizamos a partir de la siguiente enumeración. Primero hemos planteado una breve contextualización sobre el período que nos ocupa, a partir de allí inquirimos en la escritura de libros de texto escolares en manos de maestras, que intentaban auxiliar a docentes y alumnos en la tarea cotidiana llevada adelante en los salones de clase de la educación primaria. Aunque la averiguación nos dictaminó ciertas certezas, también dio lugar a preguntas a responder luego de este primer acercamiento a la temática y en futuras indagaciones: ¿quiénes fueron estas mujeres?, ¿dónde estudiaron primero y se desempeñaron en el magisterio luego?, ¿qué experiencias laborales vivenciaron?, ¿qué motivos las impulsaron a escribir sobre educación?, ¿qué requisitos, exigencias y requerimientos debieron cumplir para publicar sus obras?, ¿qué sucesos las impulsaron a asumir dichos desafíos?, ¿escribieron sólo sobre temas referentes a educación o también se involucraron, desde la escritura o no, con otros asuntos?, ¿cuáles fueron las relaciones que entablaron y con quiénes para desempeñarse dentro de las prácticas de escritura?, ¿debieron para ello renunciar al ejercicio de la docencia o lo hicieron una vez retiradas de su trabajo como maestras?, ¿qué hay detrás de cada una de sus experiencias?

Estudiar libros de maestras argentinas del período que involucra las primeras décadas del siglo XX nos posibilitó cruzar la labor desempeñada en la docencia por estas educacionistas que, gracias a dicho trabajo, pudieron emprender la osada tarea de diseñar materiales de estudio en formato de libros.

$\mathrm{Al}$ notar la prolífica cantidad de maestras involucradas en la práctica de escribir para sus alumnos/as, decidimos seleccionar un número reducido de mujeres que nos permitieron con sus obras reflexionar sobre el modo en que presentaron los materiales de estudio, la forma en que ellas como docentes aparecieron y dieron a conocer sus opiniones en los textos, las reflexiones que sobre problemas pedagógicos elaboraron.

Gracias a los aportes de Roger Chartier (1994, 2005a, 2005b, 2008) comprendimos a los procesos de escritura y lectura en íntima relación con las experiencias prescriptivas que se vuelven objeto de análisis al poseer una fuerza performativa en su búsqueda por hacer cosas con las palabras. Asimismo, y como lo hace Michelle Perrot (2008), nos preguntamos por el sujeto mujer que diseña y escribe pensando en maestras que ofician como otros intelectuales, con autonomía, producción crítica y saberes de acción, pero desde los bordes del campo intelectual: siendo maestras y mujeres (Fiorucci, 2013). Estas educadoras escribieron sobre el saber escolar, para su progreso, poniendo en escena con sus libros cuáles fueron las prácticas de lectura y escritura que generaron estos dispositivos en el espacio áulico como así también las concepciones que "docentes, escritores de textos escolares y funcionarios asumieron en torno al texto escolar" (Sardi, 2010, p.121) Porque analizando sus prólogos dilucidamos las miradas que les otorgaban estas mujeres a sus libros; usos, estilos y propuestas pedagógicas y didácticas que encontraron aquí la posibilidad de hacerse visibles. Ellas fijaron líneas de acción para el quehacer escolar, presuponiendo que los lectores —-docentes y alumnos - actuarían consecuentemente luego de trabajar con sus textos y libros de lectura.

Dijimos en las primeras líneas del escrito que la comunicación se encontraba guiada por la historia con mujeres en perspectiva de género, la historia cultural y la historia de la educación. Estudiamos al aula como lugar posible de historiar tanto en sus aspectos materiales como en sus significados, actores, experiencias (Viñao Frago, 2008). Y así, nos topamos con un grupo de maestras que, preocupadas por las prácticas, escribieron para sus alumnos/as quedando totalmente ocultas detrás de sus producciones. Así, podemos mencionar el accionar de mujeres dedicadas a la docencia que trascendieron dicha labor para escribir. Tal el caso de Gabriela Mistral, Alfonsina Storni, Herminia Brumana en la prensa, la literatura y en el ámbito de la pedagogía, como Olga Cossettini, por ejemplo.

Nuestras maestras, interesadas en los modos de aprender de sus alumnos, permanecieron invisibles detrás de sus orientaciones prácticas acerca de los saberes escolares, dispersas tras los sucesos intrincados de la dinámica escolar. Con títulos de maestras normales, estas mujeres intervinieron en el ámbito público de 
las aulas vivenciando las prácticas y escribiendo sobre ellas, pero dejaron reservada su autoría detrás de ese accionar. Los libros ocultaron a las maestras y sus condiciones de escritoras. Resultó entonces más importante el contenido de los ejemplares que difundían (para la mejora educativa) que el reconocimiento intelectual y la autoría de estas mujeres. Varias de las maestras estudiadas en la presente comunicación afirmaron en sus escritos que, luego de muchos años en la docencia, decidieron exceder el límite de las aulas para comenzar a reconocerse como escritoras y autoras de libros, asumiendo tareas propias del oficio intelectual y sintiéndose de este modo en condiciones de hacerlo por los considerables años de trabajo en las escuelas. Ellas ocuparon un sitio hasta el momento plagado por la presencia de los varones, el que no logró evidenciarlas; no supimos quiénes fueron, cuáles sus recorridos personales e intelectuales. Como decíamos, esas peculiaridades se diluyeron bajo la importancia otorgada a los textos escolares en los que las podemos ubicar (el mundo de las prácticas educativas), "obras de divulgación y acercamiento a los contenidos que se consideraban fundamentales para formar al niño como futuro ciudadano comprometido con los valores nacionales" (Sardi, 2010, p. 77).

En todos los casos nos encontramos con mujeres adheridas al ejercicio de la docencia y por tal motivo preocupadas por los avatares que en esa actividad sucedían a diario. Con la escritura de sus libros intentaron proponer soluciones a los mismos, presentando fórmulas, secretos, instrucciones y pautas a modo de prescripciones para sus colegas. Pero, y por fuera del contenido propio y coincidente de las obras, emerge una conclusión interesante y es la que refleja a todas estas maestras como autoras de libros producto de la experiencia que el trabajo les brindó. Queda un sinnúmero de aristas pendientes a indagar sobre la temática, sin embargo, con este acercamiento esperamos haber brindado aportes a la problemática que cruza los sentidos otorgados a las mujeres, la docencia y la escritura.

\section{ReFERENCIAS}

Alloatti, N. (2007). Cuentos y lecciones: textos para los niños decimonónicos en Argentina. Revista OCNOS, (3), 91-101.

Aróstegui, J. (1995). La investigación histórica: teoría y método. Barcelona. España: Editorial Crítica.

Ascolani, A. (2010). Libros de lectura en la escuela primaria argentina: civilizando al niño urbano y urbanizando al niño campesino (1900-1946). Revista Belo Horizonte, 26 (01), 303-326.

Chartier, R. (2005a). Pluma de ganso, libro de letras, ojo viajero. México: Universidad Iberoamericana. Departamento de Historia.

Chartier, R. (2005b). El pasado del presente. Escritura de la historia, historia de lo escrito. México: Universidad Iberoamericana.

Chartier, R. (2008). ¿Existe una nueva historia cultural? En S. Gayol, y M. Madero (Eds.) Formas de historia cultural (pp. 29-46). Buenos Aires: Prometeo.

Consejo Nacional de Educación (1926) El Monitor de la Educación Común. № 640- 30. Año 44. Tomo 96.

Consejo Nacional de Educación (1929). El Monitor de la Educación Común. Sección oficial. pp. 314-316.

Dussel, I. y Caruso, M. (1999). La invención del aula. Una genealogía de las formas de enseñar. Buenos Aires: Santillana.

De Nasino, Micaela (1926). Por nuestro bien. Libro de lectura para primer grado superior. (Aprobado por el Consejo Nacional de Educación) Buenos Aires: Editores Talleres Gráficos Cappellano Hermanos.

Del Prado, Alcira (1932). Sol. Texto de lectura para primer grado. Buenos Aires: Editorial Independencia.

Fiorucci, F. (2013). Presentación. Dossier: Los otros intelectuales: curas, maestros, intelectuales de pueblo, periodistas y autodidactas. Prismas. Revista de historia intelectual. 17, 165-168.

Fiorucci, F. (2016). País afeminado, proletariado feminista, mujeres inmorales e incapaces: la feminización del magisterio en disputa (1900-1920). Anuario de Historia de la Educación. 17 (2),120-137. 
Goldam, N. y Arfuch, L. (1994) Historia y prácticas culturales. Entrevista a Roger Chartier. Entre pasados. Revista de Historia, 6 (7), 133-148.

Kaufmann, C. (2012) (Coord.) Textos escolares, dictaduras y después. Miradas desde Argentina, Alemania, Brasil, España Italia. Buenos Aires: Prometeo Libros.

Lionetti, L. (2007). La misión politica de la escuela pública. Formar a los ciudadanos de la república (1870-1916). Buenos Aires: Miño y Dávila editores.

Narodowski, M. y Manolakis, L. (2001). Estado, mercado y textos escolares. Notas históricas para un modelo teórico. Revista Educación y Pedagogía. Medellín. Facultad de Educación. 13 (29-30), 27-38.

Olguín, María M. y Zamora Grondona, Virginia. (1936) Lucha. Libro de lectura para cuarto grado. Buenos Aires: Ángel Estrada y Cía.

Perrot, M. (2008). Mi historia de las mujeres. Buenos Aires: Fondo de Cultura Económica.

Puiggrós, A. (1996). Qué pasó en la educación argentina. Breve historia de la conquista hasta el menemismo. Buenos Aires: Colección triángulos pedagógicos. Kapelusz Editora.

Sardi, V. (2010). El desconcierto de la interpretación. Historia de la lectura en la escuela primaria argentina entre 1900 y 1940. Santa Fe: Universidad Nacional del Litoral. Colección ciencia y tecnología.

Solari, M. H. (1991). Historia de la Educación Argentina. Buenos Aires: Paidós.

Viñao Frago, A. (2008). La escuela y la escolaridad como objetos históricos. Facetas y problemas de la historia de la educación. Revista História da Educação, 12(25), 9-54.

Yannoulas, S. (1996). Educar. ¿Una profesión de mujeres? La feminización del normalismo y la docencia. 1870-1930. Buenos Aires: Editorial Kapelusz

\section{Notas}

1 Año 1884, durante la primera presidencia de Julio A. Roca.

2 Varones que trabajan, estudian, gobiernan y transitan por el espacio público junto a mujeres que son su complemento y, en ese rol, asumen los cuidados del hogar, la maternidad, las prácticas de beneficencia y sociabilidad públicas y demás actividades vinculadas al cuidado de los otros (mujeres: mamás o, en su excepción, maestras, enfermeras, etcétera.). Ver Lucía Lionetti (2007) o Carolina Kaufmann (2012).

\section{BY-NC-SA}

